\title{
Order versus chaos: The impact of structure on number-space associations
}

\author{
S. Cutini ${ }^{1} \cdot$ S. Aleotti ${ }^{2} \cdot$ M. G. Di Bono ${ }^{2} \cdot$ K. Priftis $^{2}$ \\ Published online: 1 July 2019 \\ (C) The Psychonomic Society, Inc. 2019
}

\begin{abstract}
The Spatial-Numerical Association of Response Codes (SNARC) effect has been observed with different stimuli, beside Arabic numerals, such as written/spoken number words, sequences of acoustic stimuli, and groups of elements. Here we investigated how the enumeration of sets of elements can be affected by the spatial configuration of the displayed stimuli with regard to the emergence of the SNARC effect. To this aim, we asked participants to perform a magnitude comparison task with structured (i.e., dice-like) and unstructured (i.e., random) patterns of rectangles. With this manipulation, we sought to explore the presence of the SNARC effect in relation to the structure of the displayed visual stimuli. The results showed that the spatial arrangement of rectangles does not impact visual enumeration processes leading to the SNARC effect. An unexpected reversal of the size effect for unstructured stimuli was also observed. We speculate that the presence of a similar SNARC effect, both with structured and unstructured stimuli, indicates the existence of a common access to the mental number line.
\end{abstract}

Keywords SNARC effect · SNA · Visual enumeration · Magnitude comparison

\section{Introduction}

The relation between numbers and space is ubiquitous in everyday life. In Western cultures, small numbers are linked to the left side of space, whereas large numbers are linked to the right side of space (Toomarian \& Hubbard, 2018). The pervasiveness of this association can be experienced in our environment. For instance, the arrangement of the numbers on our keyboard has a left-to-right orientation, and the same is true for the numbers on the speed indicator in our cars.

The relation between numbers and space has been experimentally investigated by means of studies on SpatialNumerical Associations (SNAs; Fischer \& Shaki, 2015). The Spatial-Numerical Association of Response Codes (SNARC) effect is a paradigmatic example of SNA. For instance, in magnitude comparison tasks, participants must

S. Cutini

simone.cutini@unipd.it

1 Department of Developmental Psychology, University of Padova, Via Venezia 8, 35131 Padova, Italy

2 Department of General Psychology, University of Padova, Padova, Italy judge whether a number is larger or smaller than a reference one. Participants' responses to larger numbers are consistently faster when executed on the right rather than on the left side of the space. By contrast, participants' responses to smaller numbers are faster when executed in the left side rather than in the right side of the space (Dehaene, Dupoux, \& Mehler, 1990). The SNARC effect has also been observed in parity judgment tasks, where participants must judge whether a number is odd or even (Dehaene, Bossini, \& Giraux, 1993). Thus, the SNARC effect suggests that numbers are coded along a leftto-right linear vector (i.e., the mental number line: MNL), where small numbers occupy the leftmost positions of the vector and large numbers the rightmost positions. Alternative accounts invoke the presence of polarity coding (Proctor \& Cho, 2006) for all tasks requiring the performance of speeded binary classification tasks (e.g., the SNARC effect, the linguistic markedness of response codes: MARC effect, Nuerk, Iversen, \& Willmes, 2004a). Nevertheless, in contrast to the polarity-coding account, a dissociation between the MARC and the SNARC effect has been recently reported (Di Rosa et al., 2017). Another influential theory argues that the SNARC effect might be the result of a neural overlap between the regions that process magnitudes, such as space, numbers, and time (Walsh, 2003). It should be noted, however, that these theories are not mutually exclusive. Indeed, 
recent evidence has shown that the SNARC effect is characterized by a multicomponential nature (Basso Moro, Dell'Acqua, \& Cutini, 2018).

Interestingly, the SNARC effect has been consistently replicated with negative numbers (Fischer, 2003; Shaki \& Petrusic, 2005) and with different representation formats, such as number-words (Fias, 2001; Nuerk, Kaufmann, Zoppoth, \& Willmes, 2004b). With Arabic numerals, the SNARC effect has been also observed in tasks that do not require the processing of arithmetic information, such as phoneme monitoring, where participants are asked to judge whether a phoneme is present or not in the corresponding spoken number word of the presented Arabic digit (Fias, Brysbaert, Geypens, \& d'Ydewalle, 1996). All these findings indicate that the SNARC effect is probably the most robust and widely replicated evidence of SNAs (for reviews, see Fischer \& Shaki, 2014; Hubbard, Piazza, Pinel, \& Dehaene, 2005; Wood, Nuerk, Willmes, \& Fischer, 2008).

Although Arabic numerals have been employed in the vast majority of studies on the SNARC effect, numeric information can also be conveyed by means of other numerals (e.g., Roman numerals, written number words, spoken number words, etc.). In addition, number information (e.g., numerosity) can also be conveyed by sets of perceived stimuli (e.g., three fingers, seven tones, etc.) presented in different sensory modalities (e.g., visual, tactile, or auditory). To this extent, Nuerk, Wood, and Willmes (2005) tested the SNARC effect, with different stimuli, beside Arabic numerals, such as written number words, sequences of acoustic stimuli, and sets of dots spatially arranged to resemble dice patterns. The results revealed that the activation of an abstract representation of numbers (i.e., MNL) can be elicited by using different sensory modalities. Indeed, the SNARC effect with the auditory stimuli did not differ from that found with visual stimuli. Notably, dots arranged in dice-like patterns yielded the SNARC effect in the same way that Arabic numbers did.

The core feature of dots arranged in dice-like patterns, used by Nuerk et al. (2005), was their structured spatial arrangement. Indeed, the spatial arrangement of the stimuli was characterized by symmetry and equal inter-dots distances. Note, however, that the overlearned arrangement of dice-like patterns might guide access to the MNL not by means of visual enumeration of the displayed dots (i.e., subitizing and/or estimation; Cutini, Scatturin, Basso Moro, \& Zorzi, 2014), but by means of automatic pattern recognition, in a way similar to that of Arabic digit recognition, letter (e.g., see Schmitt, 2016) and non-letter shapes (for a review, see Lachmann \& van Leeuwen, 2014).

In the present study, we extended the conceptual framework of Nuerk et al. (2005) by investigating how the spatial configuration of the displayed stimuli could influence the emergence of the SNARC effect. Hence, we presented participants with visual stimuli, constructed according to different configurational criteria. Beside the structured arrangement (i.e., dice-like patterns), we used an unstructured arrangement of the stimuli (i.e., random pattern) whose numerosity can only be processed by means of visual enumeration. With this extension, we aimed to explore the presence of the SNARC effect in relation to the structure of the displayed visual stimuli. If the presence of the SNARC effect relied uniquely on pattern recognition (e.g., the dice-like patterns), we expected to find a SNARC effect with structured stimuli, but not with unstructured stimuli. Alternatively, if the SNARC effect did not depend entirely on the structure of the visual stimuli, then the SNARC effect should be observed with both structured and unstructured stimuli, suggesting that both stimulus arrangements have access to a common MNL.

\section{Method}

\section{Participants}

Forty students (20 males, 20 females; age: $M=22.6$ years; $S D$ $=1.7$ ) from the University of Padua participated in this study. Thirty-nine participants were right-handed and one participant was left-handed. All participants had normal or corrected-tonormal visual acuity and they were naïve about the purpose of the experiment. The number of participants was defined $a$ priori, by means of the software G*POWER 3 (Power $=.95$ $\alpha=.001$, and effect size $=.25$; Faul, Erdfelder, Lang, \& Buchner, 2007). The study was approved by the institutional ethics committee.

\section{Apparatus}

The experiment was conducted on an INTEL(R) CORE(TM) 2 PC (operating system: Microsoft Windows XP Professional, version, 2002, Service Pack 3; CPU: 6320, 1,86G Hz; RAM: 1 GB), connected to a Cathodic Ray Tube monitor (screen dimension: 21-in.; colors: 32 bits; refresh rate: $100 \mathrm{~Hz}$; resolution: 1,024 x 768 pixels; video card: Intel(R) GMA 3000, NAC: Internal memory: $256 \mathrm{MB}$ ).

Participants were seated $57 \mathrm{~cm}$ from the computer screen with their head placed on a chinrest. Participants' responses were collected using two keys on a response box (https:// www.pstnet.com/hardware.cfm?ID=102) centrally positioned in front of the participants. A felt pad was applied over the right key of the response box, while the left key was left smooth. The keys were, hence, referred to as the "key with the felt pad" and "the key without the felt pad", in order to avoid the explicit expression "right-sided key" and "left-sided key" (the position of the felt pad was not counterbalanced across participants). The experiment was programmed and administered by means of E-Prime2 ProRC 8 (Version: 2.0. 8.90; Psychology Software Tools, Inc., 2012). 


\section{Stimuli}

Stimuli were a central cross (white, dimension: $0.5^{\circ}$ ) followed by a set of white rectangles (width: $0.5^{\circ}$; height: $3^{\circ}$ ), included in a $12^{\circ} \times 12^{\circ}$ area, and presented in the center of a black background screen. The rectangles varied in number size (from one to nine; five was excluded), orientation $\left(0^{\circ}\right.$ or $90^{\circ}$; this manipulation was added in order to increase variability, to avoid ceiling effects due to perceptual learning), and configuration (structured or unstructured). Stimuli were "structured" when rectangles were organized following constant and regular distances in space, similar to the dice pattern. In contrast, stimuli were "unstructured" when rectangles were organized randomly, without any regularity in the distances among them (Fig. 1).
Structured, $0^{\circ}$ orientation stimuli

Structured, $90^{\circ}$ orientation stimuli
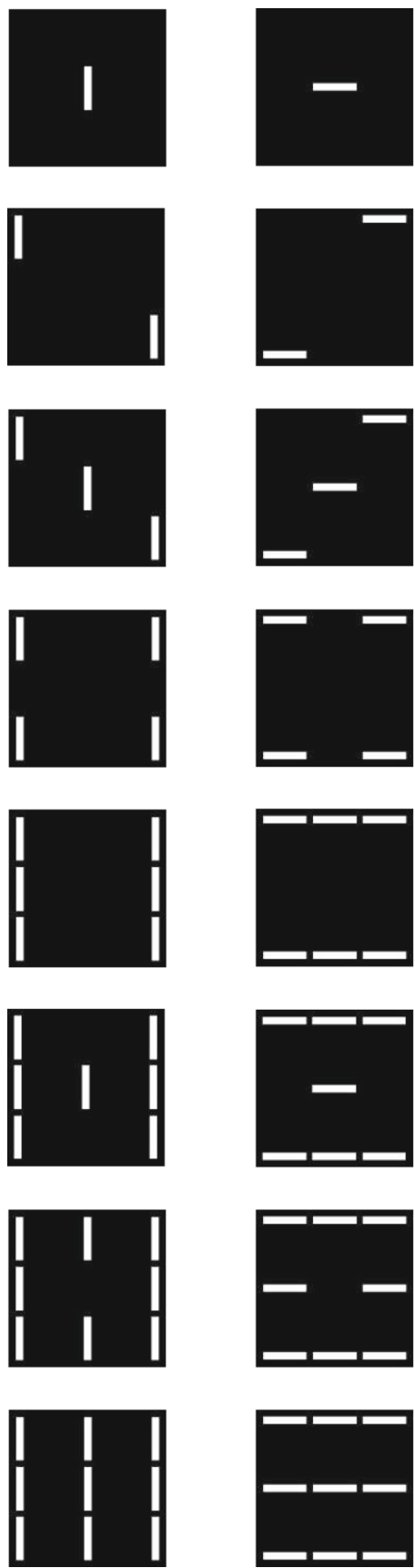

Unstructured, $0^{\circ}$ orientation stimuli
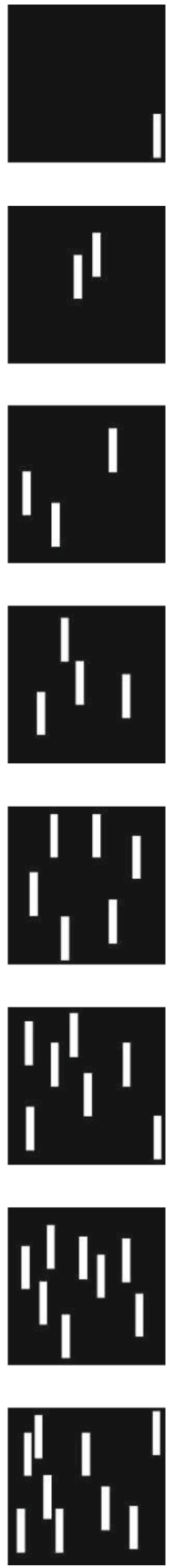
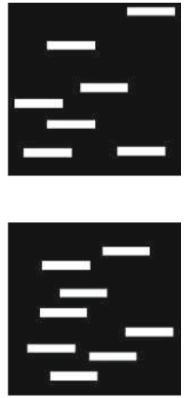

Unstructured, $90^{\circ}$ orientation stimuli
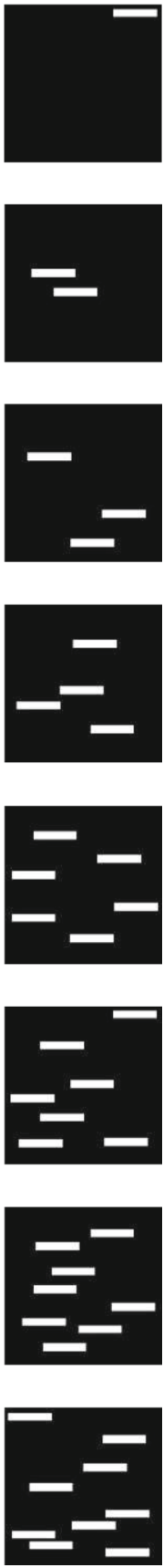

Fig. 1 The complete set of stimuli 


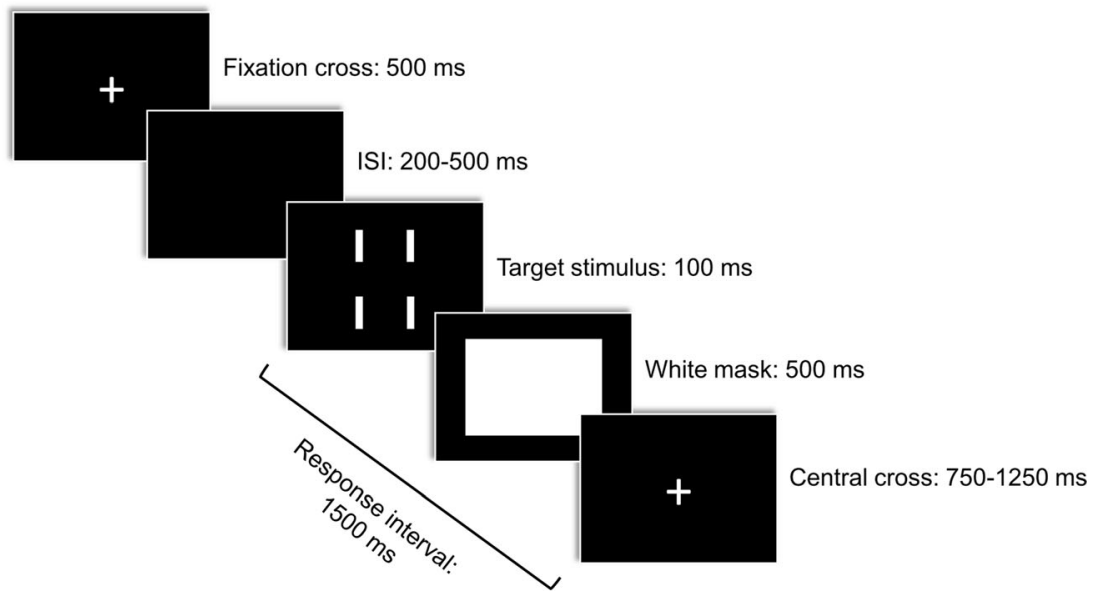

Fig. 2 Trial sequence

\section{Procedure}

Each participant was seated in front of the computer screen and his/her index fingers were placed on the respective response box's keys (right and left). Participants had to decide whether the number of rectangles was smaller or larger than the reference number (five), by pressing, as fast and accurately as possible, one of the two response keys. In the first block, the "smaller-than-five" response was assigned to the "key without felt pad" and the "larger-than-five" response to the "key with felt pad"; in the second block, the response-key assignment was reversed. The abovementioned response-key assignment was counterbalanced across participants (i.e., half of the participants performed the sequence first block $\rightarrow$ second block and half of the participants performed the sequence second block $\rightarrow$ first block).

Each trial started with the presentation of the fixation cross, lasting for $500 \mathrm{~ms}$. Then, the fixation cross disappeared and, after a variable inter-stimulus interval (ISI $=200-500 \mathrm{~ms}$ ) in order to avoid automatic responses, the target stimulus (i.e., a set of rectangles) appeared for $100 \mathrm{~ms}$. Afterwards, a white mask appeared (until the response was executed or $500 \mathrm{~ms}$ elapsed). Finally, a central cross appeared, lasting for a variable duration (i.e., 750-1,250 ms) before the next trial began. Responses were recorded from the target stimulus onset over a maximum period of 1,500 ms (Fig. 2).

\section{Design}

We used a within-participants experimental design. Independent variables were number size (two levels: small: 1-4/large: 6-9), response hand assignment (two levels: left/right), and stimulus configuration (two levels: structured/ unstructured). Therefore, independent variables were manipulated according to a 2 X 2 X 2 design. The dependent variable was the reaction time (RT) of each participant's response. Eight conditions resulted by crossing all levels of independent variables with each other. Half of them were compatible with the SNARC effect (i.e., number size smaller than five - leftkey response, and number size larger than five - right-key response), whereas the other half were incompatible (i.e., number size smaller than five - right-key response, and number size larger than five - left-key response).

The experiment consisted of two blocks of 160 trials each. The response-key assignment was counterbalanced across participants, and stimulus configuration was randomized. All participants received eight practice trials before the experimental blocks.

\section{Results}

We performed statistical analyses using IBM-SPSS for Windows (version 23). Responses on the practice trials were excluded from further analyses. The percentage of correct trials and errors was calculated for each participant. Error rate

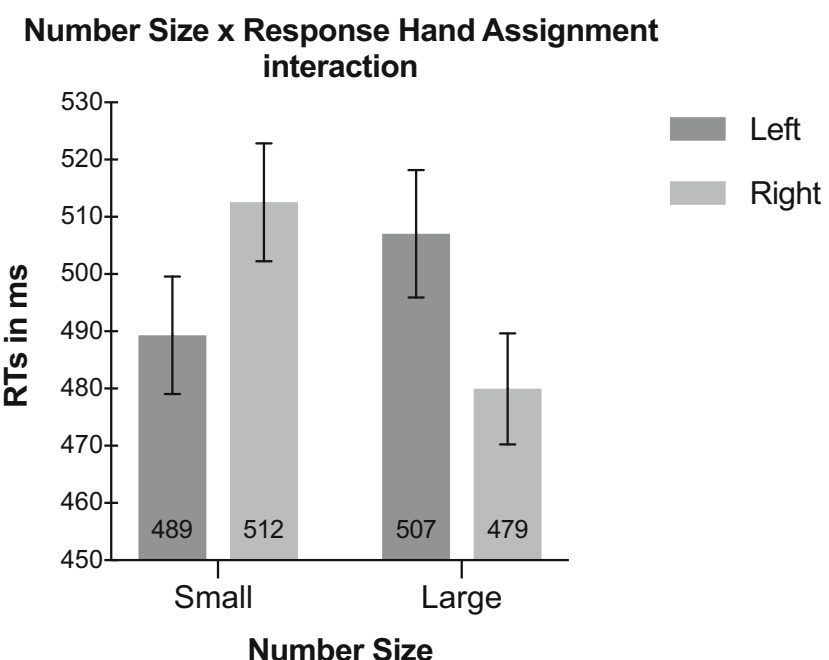

Fig. 3 The interaction Number Size X Response Hand Assignment. Error bars represent confidence intervals $(95 \%)$ corrected for withinparticipants designs (O’Brien \& Cousineau, 2014; single method) 
Table 1 Paired samples t-tests on the interaction Number Size X Response Hand Assignment

\begin{tabular}{|c|c|c|c|c|c|c|c|c|c|}
\hline \multirow[t]{2}{*}{$t$} & \multirow[t]{2}{*}{$\mathrm{df}$} & \multirow[t]{2}{*}{$p$ (one-tailed) } & \multirow[t]{2}{*}{ Mean difference } & \multirow[t]{2}{*}{ SE difference } & \multicolumn{2}{|c|}{$95 \%$ confidence interval } & \multirow{2}{*}{$\begin{array}{l}\text { Cohen's } \\
d\end{array}$} & \multicolumn{2}{|c|}{$95 \%$ confidence interva } \\
\hline & & & & & lower & upper & & lower & upper \\
\hline-1.996 & 39 & .00265 & -17.72 & 8.873 & -35.663 & .232 & -0.315 & -0.631 & .004 \\
\hline 4.001 & 39 & .00014 & 32.82 & 8.202 & 16.228 & 49.41 & .632 & .289 & .969 \\
\hline
\end{tabular}

(i.e., incorrect responses and omissions) was $4.2 \%$ of all the trials. Participants with error percentages lower than $2.5 \mathrm{SDs}$ from the mean of all participants would have been excluded from the analyses. Nevertheless, none of the participants fulfilled this criterion; thus, all participants were included in the following analyses. For correct trials only, we then calculated mean RTs and SDs, separately for each participant and each experimental condition. Finally, RTs above and below $2.5 \mathrm{SDs}$ from each participant/condition mean (i.e., $4.09 \%$ of correct trials), were excluded from further analyses.

A three-way repeated-measures analysis of variance (ANOVA) with Number Size (small/large), Response Hand Assignment (left/right), and Stimulus Configuration (structured/ unstructured) was carried out. The main effect of Number Size was significant, $F(1,39)=4.765, p=.035, \eta_{\mathrm{p}}{ }^{2}=.109$. The main effect of Response Hand Assignment was not significant, $F(1$, $39)=.439, p=.511, \eta_{\mathrm{p}}{ }^{2}=.011$, nor was the main effect of Stimulus Configuration, $F(1,39)=.601, p=.443, \eta_{\mathrm{p}}{ }^{2}=.015$.

The interaction Number Size X Response Hand Assignment was significant, indexing the SNARC effect, $F(1,39)=10.427, p=.003, \eta_{p}{ }^{2}=.211$ (Fig. 3). A priori simple effects analyses, by means of one-tailed, pairedsamples t-tests, revealed significant differences (Table 1). In particular, RTs for the small-left condition were significantly faster than RTs for the large-left condition. In addition, RTs for the large-right condition were significantly faster than RTs for the small-right condition (Table 1).

The interaction Number Size X Stimulus Configuration was significant $F(1,39)=30.987, p=.000002, \eta_{\mathrm{p}}{ }^{2}=.443$ (Fig. 4). A posteriori simple-effect analyses, by means of twotailed, paired-samples t-tests, corrected with Bonferroni, revealed significant differences (Table 2). In particular, RTs for the large-unstructured condition were significantly faster than RTs on the small-unstructured condition. In contrast, RTs for the small-structured condition were significantly faster than RTs on the large-structured condition.

The interaction Response Hand Assignment X Stimulus Configuration was not significant, $F(1,39)=1.384, p=.247$, $\eta_{\mathrm{p}}{ }^{2}=.034$, as well as the crucial interaction Number Size X Response Hand Assignment X Stimulus Configuration $F(1,39)$ $=.734, p=.397, \eta_{\mathrm{p}}{ }^{2}=.018$.

Pearson's correlation coefficient was computed to assess the relation between the SNARC effect for the structured condition and the SNARC effect for the unstructured condition. First, for each participant, the structured and unstructured conditions were considered separately. Then, the strength of the SNARC effect was tested by calculating the difference between RTs (i.e., dRT) for small numbers and RTs for large numbers, separately. For small numbers, RTs of the left hand (i.e., compatible condition with the SNARC effect) were subtracted from RTs of the right hand (i.e., incompatible condition with the SNARC effect). In contrast, for large numbers, RTs of the right hand (i.e., compatible condition with the SNARC effect) were subtracted from RTs of the left hand (i.e., incompatible condition with the SNARC effect). Afterwards, the mean dRTs for small and large numbers were calculated, for each participant, separately for the unstructured and the structured conditions. These mean dRTs were finally subjected to Pearson's correlation analysis. The positive correlation between the dRTs of the unstructured and the mean dRTs of the structured stimulus configuration was remarkably high, $r=.895, p=6.4525 \mathrm{e}-15$.

In order to establish whether the results provided evidence for or against the null hypothesis $\left(\mathrm{H}_{0}\right)$, a Bayesian factor $\left(\mathrm{BF}_{01}\right)$ hypothesis test was carried out using a prior distribution of .707. A Bayesian paired-sample t-test was performed to compare the strength of the SNARC effect between structured and unstructured conditions. The results indicated that

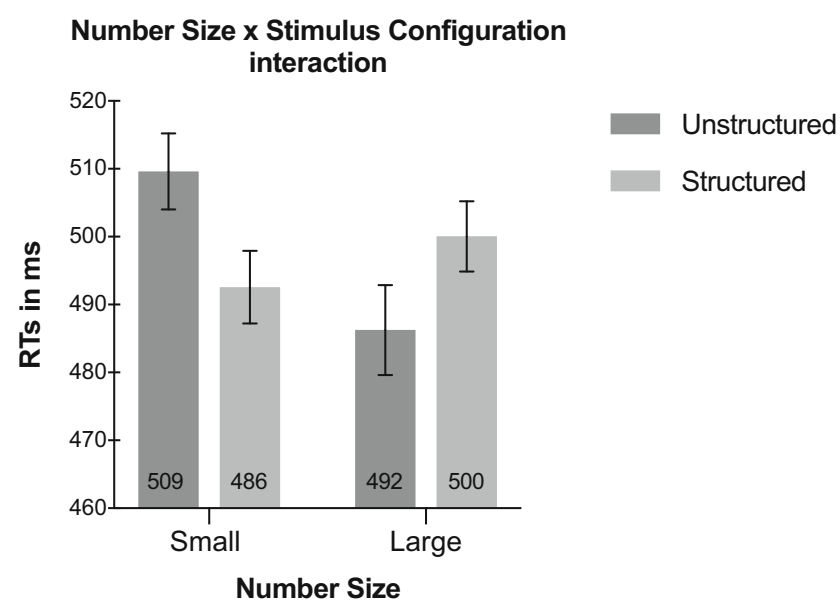

Fig. 4 The interaction Number Size X Stimulus Configuration. Error bars represent confidence intervals $(95 \%)$ corrected for within-participants designs (O’Brien \& Cousineau, 2014; single method) 
Table 2 Paired samples t-tests on the interaction Number Size X Stimulus Configuration

\begin{tabular}{|c|c|c|c|c|c|c|c|c|c|c|}
\hline & \multirow[t]{2}{*}{$t$} & \multirow[t]{2}{*}{$\mathrm{df}$} & \multirow[t]{2}{*}{$p$ (two-tailed) } & \multirow[t]{2}{*}{ Mean difference } & \multirow[t]{2}{*}{ SE difference } & \multicolumn{2}{|c|}{$95 \%$ confidence interval } & \multirow{2}{*}{$\begin{array}{l}\text { Cohen's } \\
d\end{array}$} & \multicolumn{2}{|c|}{$95 \%$ confidence interval } \\
\hline & & & & & & lower & upper & & lower & upper \\
\hline $\begin{array}{l}\text { Small-Unstructured } \\
\text { vs. Small-Structured }\end{array}$ & 5.205 & 39 & .000003 & 16.68 & 3.205 & 10.197 & 23.161 & .823 & .464 & 1.184 \\
\hline $\begin{array}{l}\text { Large-Unstructured } \\
\text { vs. Large-Structured }\end{array}$ & -3.584 & 39 & .000479 & -13.81 & 3.853 & -21.599 & -6.013 & -.567 & -0.896 & -0.228 \\
\hline
\end{tabular}

the observed data were 4.161 times more likely under $\mathrm{H}_{0}$ than under the alternative hypothesis $\left(\mathrm{H}_{1}\right.$; Table 3$)$.

Moreover, a Bayesian Pearson correlation test between structured and unstructured conditions was performed. The results were in line with the frequentist inference analysis results.

Results were further analyzed by means of a regression for repeated-measures designs (Method 3, Lorch \& Myers, 1990). For each participant, a linear regression analysis was performed, both for structured and for unstructured stimuli. The independent variable was Number (i.e., 1-9, except five), whereas the dependent variable was the difference between the RTs for the right hand and the RTs for the left hand (dRT: RTs right hand - RTs left hand; note that negative dRTs indicate faster responses with the right hand and vice versa). The betas obtained with the regression were significantly different from zero for both structured $\left(M_{\beta}=40.68, S D\right.$ $=77.11, t(39)=3.336, p=.002,95 \%$ CI $[16.02,65.34], d=$ .528 , CI $[.193, .856])$ and unstructured stimuli $\left(M_{\beta}=43.18\right.$, $S D=99.24, t(39)=2.752, p=.009,95 \%$ CI $[11.45,74.92], d$ $=.435$, CI $[.108, .757])$.

Furthermore, the betas for the structured and unstructured stimuli were strongly correlated ( $r=.801, p=5.5925 \mathrm{e}-10$; Fig. 5), consistent with the positive correlation found between dRTs of the structured stimulus configuration and dRTs of the unstructured stimulus configuration.

\section{Discussion}

Our working hypothesis stemmed from the pioneering study of Nuerk et al. (2005), who found that structured stimuli, with dots arranged in a dice-like format, elicited a SNARC effect. Furthermore, Nemeh, Humberstone, Yates, and Reeve (2018) and Zhou, Shen, Li, and Cui (2016) have reported the SNARC effect with unstructured stimuli, by means of large numerosities (i.e., arrays with numerosities of between 11 and 60 elements). Our results support and extend these findings, by testing the specific effect of stimulus arrangement,

Table 3 Bayesian Pearson's correlation results

\begin{tabular}{|c|c|c|c|c|}
\hline & & & $r$ & $\mathrm{BF}_{01}$ \\
\hline Structured & - & Unstructured & 0.895 & $4.517 e-32$ \\
\hline
\end{tabular}

with small numerosities (i.e., 1-9) that are typically used in classic SNARC paradigms. We have indeed shown that the SNARC effect is independent of the stimulus format, because both structured and unstructured stimuli elicited a consistent SNARC effect. If the SNARC effect was guided by the specific structure of the stimuli, the three-way ANOVA would have been significant. This was not the case: the effect size of the three-way non-significant interaction was very small (i.e., 0.018), and such a null effect could not be attributed to low statistical power, because our sample was a priori calculated to guarantee suitable power (.95). In the same vein, the Bayesian analysis confirmed that structured and unstructured stimuli yielded a comparable SNARC effect. As further proof, the SNARC effect for structured stimuli was remarkably correlated with that for unstructured stimuli.

On the other hand, the unexpected interaction between numerosity and stimulus configuration is difficult to interpret. For instance, it might originate from the specific spatial arrangement of the elements on each stimulus. Strikingly, while the size effect for structured stimuli shows the typical pattern observed for Arabic numerals (i.e., slower RTs as numerosity increases), we found the opposite pattern for unstructured stimuli: RTs for small numbers were slower than RTs for large numbers. This effect might be explained in terms of the peculiar aspects of the stimuli: in this vein, structured stimuli can be processed in a way similar to that of symbolic numerals

\section{Betas correlation}

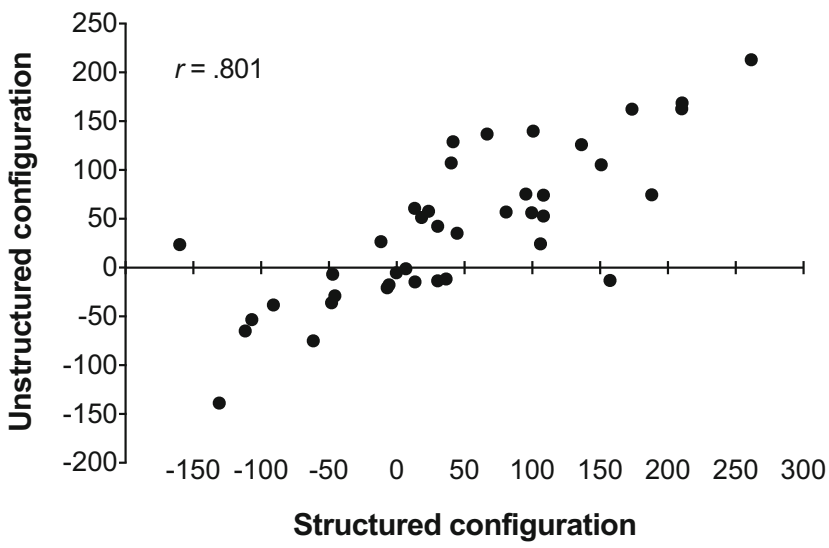

Fig. 5 Scatterplot of the correlation between the betas for unstructured and structured stimuli 
(e.g., Arabic digits), whereas unstructured stimuli do not necessarily convey a symbolic connotation. An alternative explanation might reside in the influence exerted by visual features on the perceived numerosity of the stimuli: usually, a dense arrangement of the elements may trigger an overestimation while a sparse arrangement elicits an underestimation of numerosity (Gebuis \& Reynvoet, 2012). Since unstructured stimuli are characterized by more densely arranged elements than structured ones (see Fig. 1), unstructured stimuli with large numerosity should yield faster RTs for the right hand than for the left hand (and vice versa for structured stimuli with low numerosity). Although this speculation is consistent with the present results, it deserves to be further investigated in future studies, in which the spatial arrangement of the stimuli should be further manipulated.

In conclusion, in the present study we highlighted for the first time that the spatial arrangement and structure of the array of stimuli does not impact visual enumeration processes leading to the SNARC effect, since we found that familiarity with the stimuli (i.e., dice), does not seem to influence the access to the MNL. Thus, we speculate that the presence of a similar SNARC effect, both with structured and with unstructured stimuli, indicates the existence of common access to the MNL.

\section{Compliance with ethical standards}

Conflict of interest All the authors declare that they have no conflicts of interest.

Ethical approval All procedures performed in studies involving human participants were in accordance with the ethical standards of the institutional and/or national research committee and with the 1964 Helsinki declaration and its later amendments or comparable ethical standards.

Informed consent Informed consent was obtained from all individual participants included in the study.

\section{References}

Basso Moro, S., Dell'Acqua, R., \& Cutini, S. (2018). The SNARC effect is not a unitary phenomenon. Psychonomic Bulletin \& Review, 25, 688-695. https://doi.org/10.3758/s13423-017-1408-3

Cutini, S., Scatturin, P., Basso Moro, S., \& Zorzi, M. (2014). Are the neural correlates of subitizing and estimation dissociable? An fNIRS investigation. NeuroImage, 85, 391-399. https://doi.org/10. 1016/j.neuroimage.2013.08.027

Dehaene, S., Bossini, S., \& Giraux, P. (1993). The mental representation of parity and number magnitude. Journal of Experimental Psychology: General, 122, 371-396. https://doi.org/10.1037/00963445.122.3.371

Dehaene, S., Dupoux, E., \& Mehler, J. (1990). Is numerical comparison digital? Analogical and symbolic effects in two-digit number comparison. Journal of Experimental Psychology: Human Perception and Performance, 16, 626-641. https://doi.org/10.1037/0096-1523. 16.3 .626
Di Rosa, E., Bardi, L., Umiltà, C., Masina, F., Forgione, M., \& Mapelli, D. (2017). Transcranial direct current stimulation (tDCS) reveals a dissociation between SNARC and MARC effects: Implication for the polarity correspondence account. Cortex, 93, 68-78. https://doi. org/10.1016/j.cortex.2017.05.002

Faul, F., Erdfelder, E., Lang, A.-G., \& Buchner, A. (2007). G*Power 3: A flexible statistical power analysis program for the social, behavioral, and biomedical sciences. Behavior Research Methods, 39, 175-191. https://doi.org/10.1016/j.neuroimage.2013.08.027

Fias, W. (2001). Two routes for the processing of verbal numbers: Evidence from the SNARC effect. Psychological Research, 65, 250-259. https://doi.org/10.1007/s004260100065

Fias, W., Brysbaert, M., Geypens, F., \& d'Ydewalle, G. (1996). The importance of magnitude information in numerical processing: Evidence from the SNARC Effect. Mathematical Cognition, 2, 95110. https://doi.org/10.1080/135467996387552

Fischer, M. H. (2003). Cognitive representation of negative numbers. Psychological Science, 14, 278-282. https://doi.org/10.1111/14679280.03435

Fischer, M. H., \& Shaki, S. (2014). Spatial associations in numerical cognition-From single digits to arithmetic. The Quarterly Journal of Experimental Psychology, 67, 1461-1483. https://doi. org/10.1080/13506280244000186

Fischer, M. H., \& Shaki, S. (2015). Two steps to space for numbers. Frontiers in Psychology, 6, 2-3. https://doi.org/10.3389/fpsyg. 2015.00612

Gebuis, T., \& Reynvoet, B. (2012). The role of visual information in numerosity estimation, PloSOne, 7(5), e37426. https://doi.org/10. 1371/journal.pone.0037426

Hubbard, E., Piazza, M., Pinel, P., \& Dehaene, S. (2005). Interactions between number and space in parietal cortex. Nature Reviews Neuroscience, 6, 435-438. https://doi.org/10.1038/nrn1684

Lachmann, T., \& van Leeuwen, C. (2014). Reading as functional coordination: not recycling but a novel synthesis. Frontiers in Psychology, 5, 1046. https://doi.org/10.3389/fpsyg.2014.01046

Lorch, R. F., \& Myers, J. L. (1990). Regression analyses of repeated measures data in cognitive research. Journal of Experimental Psychology: Learning, Memory, and Cognition, 16, 149. https:// doi.org/10.1037/0278-7393.16.1.149

Nemeh, F., Humberstone, J., Yates, M. J., \& Revee, R. A. (2018). Nonsymbolic magnitudes are represented spatially: Evidence from a non-symbolic SNARC task. PloS one, 13, e0203019. https://doi. org/10.1371/journal.pone.0203019

Nuerk, H.-C., Iversen, W., \& Willmes, K. (2004a). Notational modulation of the SNARC and the MARC (linguistic markedness of response codes) effect. The Quarterly Journal of Experimental Psychology. A, Human Experimental Psychology, 57, 835-863. https://doi.org/10. 1080/02724980343000512

Nuerk, H. C., Kaufmann, L., Zoppoth, S., \& Willmes, K. (2004b). On the development of the mental number line: More, less, or never holistic with increasing age? Developmental Psychology, 40, 1199-1211. https://doi.org/10.1037/0012-1649.40.6.1199

Nuerk, H. C., Wood, G., \& Willmes, K. (2005). The universal SNARC effect: The association between number magnitude and space is amodal. Experimental Psychology, 52, 187-194. https://doi.org/10. 1027/1618-3169.52.3.187

O'Brien, F., \& Cousineau, D. (2014). Representing error bars in withinsubject designs in typical software packages. The Quantitative Methods for Psychology, 10, 56-67. https://doi.org/10.20982/tqmp. 10.1.p056

Proctor, R. W., \& Cho, Y. S. (2006). Polarity correspondence: A general principle for performance of speeded binary classification tasks. Psychological Bulletin, 132, 416-442. https://doi.org/10.1037/ 0033-2909.132.3.416 
Schmitt, A. (2016). What makes a letter a letter? - New evidence for letter-specific processing strategies. Dissertation. TU Kaiserslautern, Germany.

Shaki, S., \& Petrusic, W. M. (2005). On the mental representation of negative numbers: Context-dependent SNARC effects with comparative judgments. Psychonomic Bulletin \& Review, 12, 931-937. https://doi.org/10.3758/BF03196788

Psychology Software Tools, Inc. (2012). E-Prime2 ProRC 8 (Version: 2.0.8.90) [Computer software]. Retrieved from http://www.pstnet. com/eprime/cfm

Toomarian, E. Y., \& Hubbard, E. M. (2018). On the genesis of spatialnumerical associations: Evolutionary and cultural factors coconstruct the mental number line. Neuroscience and Biobehavioral Reviews, 90, 184-199. https://doi.org/10.1016/j.neubiorev.2018.04. 010

Walsh, V. (2003). A theory of magnitude: common cortical metrics of time, space and quantity. Trends in Cognitive Sciences, 7, 483-488. https://doi.org/10.1016/j.tics.2003.09.002
Wood, G., Willmes, K., Nuerk, H. C., \& Fischer, M. H. (2008). On the cognitive link between space and number: A meta-analysis of the SNARC effect. Psychology Science Quarterly, 50, 489-525.

Zhou, X., Shen, C., Li, L., Li, D., \& Cui, J. (2016). Mental Numerosity Line in the Human's Approximate Number System. Experimental Psychology, 63, 169-179. https://doi.org/10.1027/1618-3169/ a000324

Open Practice StatementThe study was not pre-registered. Data and material will be made available upon request.

Publisher's note Springer Nature remains neutral with regard to jurisdictional claims in published maps and institutional affiliations. 